\title{
Homöopathische Behandlung der Neurodermitis
}

\section{Besonderheiten und Fallbeispiele mit Polaritätsanalyse}

\author{
Von Heiner Frei
}

\section{Zusammenfassung}

Die Neurodermitis ist eine häufige Erkrankung bei Kindern, die konventionell medizinisch nur unterdrückt, aber nicht geheilt werden kann. Die Folge sind in der Regel innere Allergien wie Heuschnupfen und Asthma. Mit der Homöopathie besteht dagegen eine Möglichkeit, das Leiden definitiv zu heilen. Solange nur Hautsymptome vorliegen, ist die Bestimmung des Arzneimittels schwierig, weil die Symptomatik auch in einem übertragenen Sinne oberflächlich ist. Kann man innere Symptome zur Mittelbestimmung heranziehen, so steigen die Erfolgschancen deutlich.

In diesem Artikel wird einleitend die Polaritätsanalyse vorgestellt und anhand von zwei Neurodermitis-Patienten erläutert. Beim ersten handelt es sich um einen Säugling, der alle möglichen Heilungshindernisse aufwies, beim zweiten um ein größeres Kind mit inneren Nebensymptomen, die direkt zum homöopathischen Arzneimittel führten. Abschließend wird der Umgang mit möglichen Fehlerquellen diskutiert.
\end{abstract}

\section{Schlüsselwörter}

Neurodermitis, Homöopathie, Polaritätsanalyse, Hautsymptome, innere Symptome.

\section{Summary}

Neurodermitis is a frequent disease in children. Conventional medicine can only suppress its symptoms and is not able to cure it. In consequence the patients often develop inner allergies such as hayfever or asthma. Contrarily homeopathy is able to heal these patients. But as long as they have only skin symptoms, the identification of the homeopathic remedy is not easy, because skin symptoms are superficial. If the patients have internal symptoms remedy determination becomes much more precise and efficient. In this article we introduce Polarity Analysis, a new method for remedy determination, and explain it with two case histories of neurodermitis patients. The first one is an infant, who showed many possible healing obstacles, the second an older child with concomitant internal symptoms that led directly to the homeopathic remedy. Finally we discuss possible pittfalls in neurodermitis treatment of small children.

\section{Keywords}

Neurodermitis, Homeopathy, Polarity Analysis, Skin Symptoms, Internal Symptoms.

\section{Einführung}

An Neurodermitis leiden 10-15\% der Vorschulkinder und ca. 3\% der Erwachsenen. Das klinische Erscheinungsbild ist eine lokale oder generalisierte Rötung mit Verdickung und Felderung der Haut. Die Hautausschläge können trocken, schuppend oder nässend sein. Typisch ist ein Befall der Gelenkregionen, oft mit Juckreiz. Es besteht eine familiäre Disposition, trockene Luft und Stress wirken begünstigend. Häufigste Ursache der Neurodermitis ist eine allergische Reaktion auf eine oder mehrere Substanzen, wobei alles infrage kommt, mit dem die Patienten Kontakt haben. Häufige Allergene sind Nahrungsmittel, Hausstaubmilben, Tierhaare und Pollen.

Bei der Behandlung der Neurodermitis ist in erster Linie die gute Hautpflege wichtig. Schulmedizinisch werden Corticosteroide eingesetzt, die eine Linderung bewirken, solange man sie anwendet, bei längerer Therapie besonders bei Kindern aber zu systemischen Nebenwirkungen führen können: Mit der Zeit sprechen die Patienten immer weniger darauf an, weshalb die Lösung nicht befriedigt. Homöopathisch kann die Neurodermitis oft geheilt werden, aber die Mittelfindung ist in der Regel nicht einfach.

\section{Besonderheiten der homöopathischen Neurodermitis-Behandlung}

\section{Bewährte Indikationen}

Bei akuten Ekzemen können wir die Behandlung mit einer Dosis Belladonna C 200 beginnen. Bessert diese nicht innerhalb von 10 Tagen, so folgt Sulfur C 30. Nach unserer Erfahrung kommt es mit diesem Vorgehen bei ungefähr einem Drittel der Patienten zu einer Besserung, manchmal sogar zu einer Heilung. Ist die Neurodermitis nach einer Impfung aufgetreten, so sind die spezifischen Impfnosoden oft erfolgreich, also z.B. Iso-Infanrix-Quinta 
C 200, Iso-Prévenar-13 C 200 oder Iso-Neisvac-C C 200 (von Schmidt Nagel).

\section{Individuelle Mittelbestimmung}

Verfehlen bewährte Indikationen ihren Zweck, so ist die individuelle homöopathische Mittelbestimmung der nächste Schritt. Dabei ist zu beachten, dass eine Mittelbestimmung mit Hautsymptomen auch in einem übertragenen Sinn oberflächlich und die Erfolgsquote entsprechend klein ist. Optimal wäre die Repertorisation innerer Begleitsymptome, z.B. von Verdauungsproblemen oder Atemwegserkrankungen. Meistens ist die Neurodermitis eine einseitige, oligosymptomatische Erkrankung. Wir brauchen deshalb eine Methode der Mittelbestimmung, die diese Schwierigkeit bewältigen kann. Die Polaritätsanalyse hat sich dazu als besonders geeignet erwiesen. In diesem Artikel wird zunächst die Methode beschrieben und danach anhand zweier Neurodermitis-Fälle vorgestellt.

\section{Dosierung bei individueller Behandllung von Hauterkrankungen}

Aufgrund des meist hohen Leidensdrucks der Patienten empfiehlt es sich, zunächst die Reaktion des Patienten mit einer Dosis des bestpassenden Mittels in der Potenz C 30 zu testen. Kommt es nicht zu einer erheblichen Verschlimmerung, kann nach zwei Wochen eine C 200 verabreicht werden. Bei Verschlimmerungen mit nachfolgender Besserung empfiehlt es sich, die weiteren Dosen über 3-5 Becher verdünnt zu verabreichen (Details siehe www.heinerfrei.ch/Praxis/Ressourcen/Verdünnen von Einzeldosen).

\section{Die Polaritätsanalyse}

Die Polaritätsanalyse (PA) ist ein exakt definiertes homöopathisches Vorgehen, das die Zuverlässigkeit der Mittelbestimmungen verbessert [6]. Sie basiert auf der Gradierung von Clemens von Bönnighausens Therapeutischem Taschenbuch, einem der ersten Repertorien der Homöopathie [3], und besteht aus 5 Elementen, die wir hier kurz erläutern wollen.

Erstes Element ist der Begriff des Genius der Arzneien, der auf Bönninghausen und Constantin Hering zurückgeht. Die Charakteristik, der Genius einer Arznei besteht aus denjenigen Symptomen, die sich in der Prüfung öfters und in verschiedenen Lokalisationen zeigten, und die auch klinisch bestätigt wurden. Es ist die Symptomenkonstellation, mit der sich ein Arzneimittel von andern Arzneien unterscheidet. Geniussymptome sind im BTB durch eine hohe Gradierung hervorgehoben (Grad 35). Wenn wir erfolgreich behandeln wollen, so müssen die charakteristischen Patientensymptome möglichst gut mit dem Genius eines Arzneimittels in Übereinstimmung gebracht werden $[2 ; 11]$.

Zweites Element sind die Modalitäten. Im Organon $\S 133$ schreibt Samuel Hahnemann, dass sich das Eigentümliche und Charakteristische eines Symptomes in dessen Modalitäten zeigt [10]. Verknüpfen wir diese Aussage mit dem Organon § 153, der besagt, dass die homöopathische Mittelwahl ganz besonders nach den eigenheitlichen, charakteristischen Symptomen der Arzneimittel ausgerichtet werden soll, so bedeutet dies nach unserer Erfahrung, dass die Übereinstimmung von Patientensymptomatik und Arzneimittel ganz besonders die Modalitäten betreffen muss.

Drittes Element sind die polaren Symptome: Das sind Symptome, die einen Gegenpol haben, wie Durst/Durstlosigkeit, Kälte verschlimmert/Kälte bessert, Verlangen nach freier Luft/Abneigung gegen freie Luft. In Bönninghausens Therapeutischem Taschenbuch finden sich ungefähr 200 polare Symptome. Die meisten davon sind Modalitäten und daher besonders wichtig bei der Arzneimittelbestimmung. Ihre Bedeutung wird noch dadurch gesteigert, dass sie praktisch immer eindeutig sind und (im Gegensatz zu den Gemütssymptomen) nicht interpretiert werden können. Sie haben deshalb eine hohe $\mathrm{Zu}-$ verlässigkeit in der Mittelbestimmung [7].

Viertes Element sind die Kontraindikationen. Bönninghausen war bestrebt, die wichtigen Symptome seiner Patienten mit dem Genius eines homöopathischen Arzneimittels widerspruchsfrei abzudecken [2]. Was bedeutet das? Ein Widerspruch zum Genius kann bei den polaren Symptomen auftreten: Der Patient kann immer nur einen Pol eines polaren Symptoms aufweisen, er kann zum Beispiel nicht gleichzeitig durstig und durstlos sein. Bei den Arzneimitteln ist das anders: Da deren Symptomatik durch mehrere Prüfer eruiert wurde, weisen sie oft beide Pole solcher Symptome auf. Meistens ist aber einer für das Mittel charakteristisch (und deshalb hoch gradiert), der andere eher ein Zufallsbefund (mit tiefer Gradierung). Bei einer vollkommenen Übereinstimmung zwischen der Patientensymptomatik und dem Genius des Arzneimittels deckt dieses die polaren Symptome hochgradig ab. Steht umgekehrt ein polares Patientensymptom in einem tiefen, der Gegenpol aber in einem hohen Grad, so entspricht der Genius des Arzneimittels nicht in allen Teilen der Patientensymptomatik. Bönninghausen bezeichnete dies als Widerspruch. Nach seiner Erfahrung erfolgte in solchen Konstellationen kaum je eine Heilung, weshalb er sie als Kontraindikation betrachtete.

Fünftes Element ist die Berechnung der Polaritätsdifferenz [6]. Dazu werden bei jedem Arzneimittel die Grade aller polaren Patientensymptome addiert. Vom Resultat wird die Summe der Grade der entsprechenden Gegenpole subtrahiert. Das Resultat ergibt einen Hinweis, wie spezifisch ein Arzneimittel die Patientensymptomatik ab- 


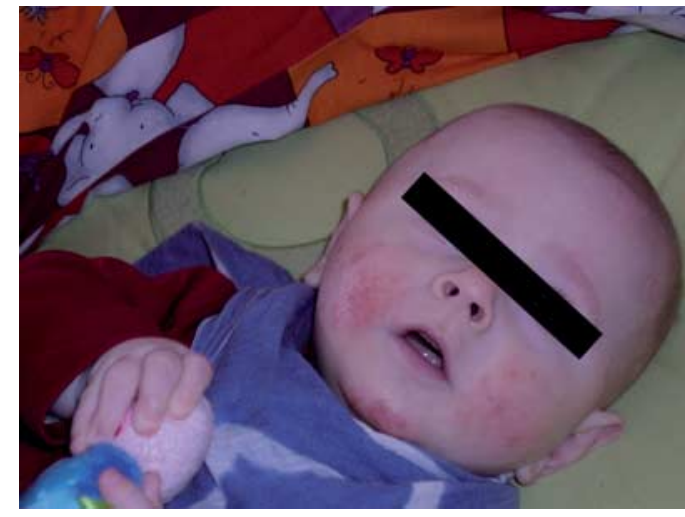

Abb. 1: Simon vor der Behandlung.

deckt: Je höher die Polaritätsdifferenz, umso besser entspricht das Arzneimittel der charakteristischen Patientensymptomatik. Die Polaritätsdifferenz beziffert also die Heilungswahrscheinlichkeit, die ein Mittel für die Krankheit des Patienten aufweist. Zu deren Berechnung sollten, wenn möglich, mindestens 5 polare Symptome verwendet werden. Um diese bei der Fallaufnahme vollständig zu erfassen, ergänzen wir die homöopathische Anamnese bei akuten Erkrankungen mit Checklisten, bei chronischen mit ausführlicheren Fragebögen. Auf diesen unterstreichen die Patienten die Symptome, die sie beobachtet haben (Download: www.heinerfrei.ch) [7].

Die Polaritätsanalyse ist während der schweizerischen ADHS-Doppelblindstudie entwickelt worden, um die Treffsicherheit der Verordnungen zu erhöhen. Durch Umsetzung der Erkenntnisse in einem Computerprogramm wurde die Analyse einfach und schnell [1]. Damit gelang auch der Nachweis eines signifikanten Unterschieds zwischen Placebo und hochpotenzierten homöopathischen Arzneimitteln [9]. Natürlich haben wir die Methode anschließend auf akute, chronische und komplexe Erkrankungen übertragen. Deren Evaluation ergab ausnahmslos eine Verbesserung der Ergebnisse [5; 6; 8]. Das praktische Vorgehen wird nachfolgend erläutert.

\section{Fallbeispiel 1}

Simon wird nach einer unauffälligen Schwangerschaft am Termin geboren. Schon in den ersten Lebenstagen beginnt ein Ausschlag im Gesicht, der sich zunehmend verschlimmert. Mit 6 Wochen geht er in gelbliche, nässende Krusten über, die typisch sind für die Säuglingsneurodermitis.

Da andere Symptome fehlen, machen wir zunächst einen Versuch mit Belladonna C 200, danach Sulfur C 30, die beide leider nichts ändern. Simon ist hypoton, blass, pastös und schwitzt stark, alles Hinweise auf Calcium carbonicum, aber auch dieses Mittel bleibt ohne Wirkung.
Impfnosoden kommen nicht in Betracht, weil er noch nicht geimpft ist. Unterdessen breitet sich das Ekzem auf den ganzen Körper aus und wird sehr schlimm, so dass sich eine individuelle Mittelbestimmung aufdrängt.

Auf dem Fragebogen für Nebensymptome, den wir bei Hauterkrankungen immer verwenden, um möglichst innere Symptome zu erfassen, unterstreicht die Mutter nur Hautsymptome ( $\mathrm{P}=$ Polare Symptome):

•. Hautausschlag nässend, schuppend, juckend

$\bullet<$ Kälte-P

$\bullet>$ Warmeinhüllen-P

$\bullet<$ beim Erwachen-P

.. > im Freien-P

.. Bewegung-P

.. $<$ in der Sonne

\section{Repertorisation, Interpretation und} Materia-medica-Vergleich [11]

Aufgrund der kargen Symptomatik verwenden wir alles zur Repertorisation, auch den Hautausschlag (••Abb. 2).

Fünf Arzneimittel decken alles ab; nur Graphites und Bryonia haben keine Kontraindikationen.

\section{Graphites [12]:}

Ekzem mit reichlicher, seröser Absonderung; bei blonden Menschen, die zu Fettsucht neigen. Ausschlag hinter den Ohren, an verschiedenen Teilen, aus dem eine wässrige, transparente, klebrige Flüssigkeit aussickert. Heftiges Jucken und Brennen.

\section{Bryonia [12]:}

Am ganzen Körper trockener und juckender Ausschlag, ...mit Jucken zum blutig Kratzen.

\section{Mittelgabe und Verlauf}

Simon erhält aufgrund der hohen Polaritätsdifferenz und der höheren Stimmigkeit im Materia-medica-Vergleich eine Dosis Graphites C 30. Zunächst tritt eine leichte Verschlechterung ein, danach eine mäßige Besserung. Die Eltern melden sich erst nach zwei Monaten wieder, weil sich die Haut erneut verschlechtert.

Mit Graphites C 200 kommt es zu einer deutlichen Besserung, die 4 Wochen andauert, dann etwas nachlässt. Graphites M und XM bringen das Ekzem fast völlig zum Verschwinden, und die Haut hält sich fortan ziemlich ruhig ( • Abb. 3).

Mit 2 1/2 Jahren erkrankt Simon an einer Scharlachangina, welche mit Aconitum C 200 wieder abheilt. Kurz darauf tritt eine Urtikaria auf (•• Abb. 4), danach beginnen Heuschnupfen und Asthma. Was ist passiert? Wahrscheinlich hat Aconitum Graphites antidotiert und zu einer Verschiebung der Symptomatik in die Tiefe geführt. Der Vorteil für uns liegt darin, dass jetzt eine innere Symptomatik vorliegt und wir eine zuverlässigere Mittelbestimmung durchführen können. 
S. A.

Neurodermitis

\begin{tabular}{|c|c|c|c|c|c|c|c|c|c|c|}
\hline \multicolumn{3}{|c|}{$+\frac{1}{1+2}$} & Graph. & Bell. & Bry. & Nat-c. & Lach. & Hep. & cic. & Ars. \\
\hline \multicolumn{3}{|l|}{ Hits } & 7 & 7 & 7 & 7 & 7 & 6 & 6 & 6 \\
\hline \multicolumn{3}{|c|}{ Sums } & 21 & 17 & 15 & 15 & 16 & 18 & 13 & 15 \\
\hline \multicolumn{3}{|c|}{ Polarity Difference } & 13 & 7 & 6 & 2 & 1 & 11 & 9 & 8 \\
\hline 90 & < Kălte allg. & P & 2 & 3 & 2 & 2 & 2 & 4 & 3 & 4 \\
\hline 56 & > Warmeinhüllen & $\mathbf{P}$ & 2 & 2 & 1 & 2 & 2 & 4 & 3 & 3 \\
\hline 111 & < Beim Erwachen & $\mathbf{P}$ & 5 & 3 & 2 & 4 & 2 & 4 & 1 & 5 \\
\hline 93 & $>$ Im Freien & $P$ & 3 & 1 & 2 & 1 & 3 & 1 & 2 & 1 \\
\hline 126 & < Bewegung, während & $\mathbf{P}$ & 3 & 4 & 4 & 1 & 1 & 3 & 2 & 1 \\
\hline 23 & < Sonne & & 2 & 2 & 3 & 4 & 3 & & & \\
\hline 44 & Hautausschlag nässend & & 4 & 2 & 1 & 1 & 3 & 2 & 2 & 1 \\
\hline 73 & > Kälte allg. & & 1 & 1 & 1 & 1 & 1 & 1 & & \\
\hline 37 & $<$ Warmeinhüllen & & & & 1 & & 1 & & & \\
\hline 28 & > Erwachen, beim & & & & 1 & 1 & 1 & & & 3 \\
\hline 110 & $<$ Im Freien & & 1 & $4 / \mathrm{Cl}$ & 1 & 2 & $4(\mathrm{Cl})$ & $3 / \mathrm{Cl}$ & 1 & 1 \\
\hline 102 & > Bewegung, während & & & 1 & 1 & $4 / \mathrm{Cl}$ & 2 & 1 & 1 & 2 \\
\hline
\end{tabular}

Abb. 2: Erste Repertorisation zu Simon [1].

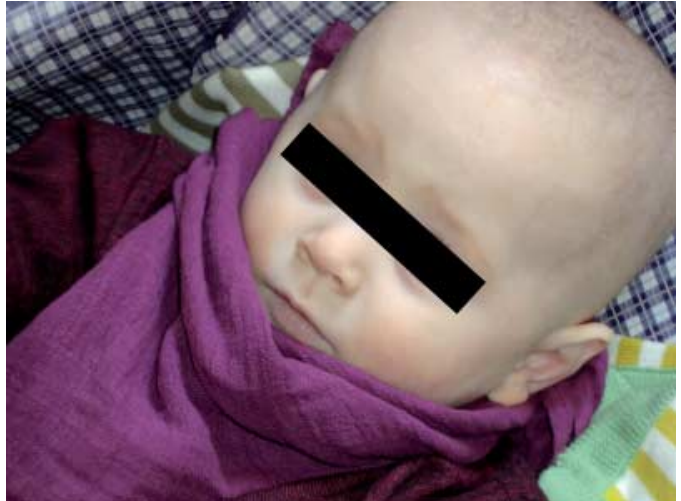

Abb. 3: Simon nach der Graphites-Gabe.

Wir machen eine neue Fallaufnahme mit den Fragebögen für HNO- und Augenerkrankungen und für Atemwege. Die Mutter bringt folgende Symptome mit:

.• Atem schnell-P

•. < Ausatmen-P

.. < Anstrengung körperlich-P

•• < Wärme-P

$\bullet>$ Entblößung-P

$\bullet>$ im Freien-P

• Durstlosigkeit-P

- Traurigkeit-P

•• Schnupfen wässrig

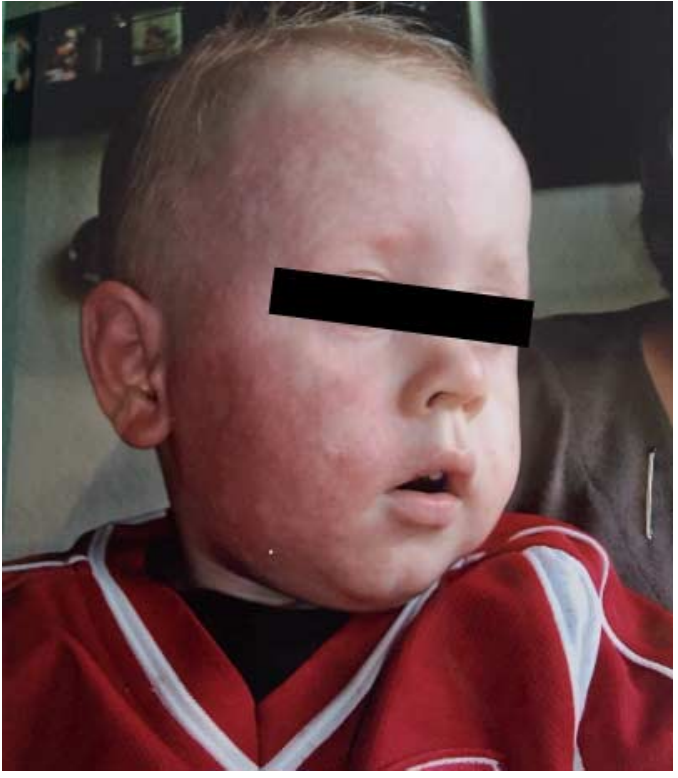

Abb. 4: Urtikaria.

Jetzt verwenden wir nur die polaren Symptome zur Repertorisation, weil sie eine genügende Differenzierung der Arzneimittel ermöglichen. 


\begin{tabular}{|c|c|c|c|c|c|c|c|c|c|c|c|c|c|}
\hline & & & Puls. & Verat. & Spig. & Staph. & Sep. & Bry. & Rhus. & Ign. & Nux-v. & Aur. & Lyc. \\
\hline \multicolumn{3}{|l|}{ Hits } & 8 & 8 & 8 & 8 & 8 & 8 & 8 & 8 & 8 & 8 & 7 \\
\hline \multicolumn{3}{|c|}{ Sums } & 25 & 19 & 16 & 14 & 17 & 16 & 16 & 16 & 15 & 12 & 21 \\
\hline \multicolumn{3}{|c|}{ Polarity Difference } & 16 & 10 & 8 & 6 & 5 & 1 & -2 & -1 & -1 & -1 & 14 \\
\hline 92 & Atem schnell & p & 3 & 3 & 2 & 1 & 4 & 3 & 3 & 3 & 3 & 2 & 4 \\
\hline 52 & < Ausatmen, beim & P & 4 & 2 & 4 & 3 & 3 & 2 & 1 & 3 & 2 & 1 & \\
\hline 70 & < Anstrengung des Körpers & P & 1 & 4 & 1 & 1 & 2 & 4 & 4 & 1 & 3 & 2 & 5 \\
\hline 73 & < Wărme allg. & $\mathbf{P}$ & 4 & 1 & 1 & 1 & 1 & 1 & 1 & 1 & 1 & 1 & 2 \\
\hline 37 & $>$ Entblößung & $\mathbf{P}$ & 2 & 3 & 3 & 2 & 1 & 1 & 1 & 2 & 1 & 1 & 4 \\
\hline 93 & $>$ Im Freien & P & 4 & 2 & 1 & 1 & 1 & 2 & 1 & 1 & 1 & 2 & 2 \\
\hline 86 & Durstlosigkeit & $\mathbf{p}$ & 4 & 2 & 3 & 3 & 3 & 1 & 2 & 1 & 2 & 1 & 1 \\
\hline 61 & Traurigkeit, Niedergeschlagenheit, We & $\mathbf{P}$ & 3 & 2 & 1 & 2 & 2 & 2 & 3 & 4 & 2 & 2 & 3 \\
\hline 63 & Atem langsam & & 1 & & & 1 & & 3 & 1 & 3 & 2 & 2 & \\
\hline 61 & $>$ Ausatmen, beim & & & 1 & 1 & & 1 & $4 / \mathrm{Cl}$ & $4 / \mathrm{Cl}$ & & & & 2 \\
\hline 6 & > Anstrengung des Körpers & & & & & & $4 / \mathrm{Cl}$ & & & $3 / \mathrm{Cl}$ & & & \\
\hline 90 & > Wărme allg. & & 1 & 1 & 2 & 2 & 2 & 2 & $4 / \mathrm{Cl}$ & $3 / \mathrm{Cl}$ & $4 / \mathrm{Cl}$ & $3 / \mathrm{Cl}$ & 1 \\
\hline 56 & $<$ Entbloßung & & 1 & & & 2 & 2 & 1 & $4 / \mathrm{Cl}$ & 1 & $3 / \mathrm{Cl}$ & $3 / \mathrm{Cl}$ & \\
\hline 110 & $<$ Im Freien & & 1 & 1 & $3 / \mathrm{Cl}$ & 2 & 1 & 1 & 2 & $3 / \mathrm{Cl}$ & $4 / \mathrm{Cl}$ & 1 & 1 \\
\hline 99 & Durst & & 2 & $3 / \mathrm{Cl}$ & 1 & 1 & 2 & $4 / \mathrm{Cl}$ & $3 / \mathrm{Cl}$ & 2 & $3 / \mathrm{Cl}$ & 1 & 1 \\
\hline 42 & Fröhlichkeit (ungewöhnlich) & & 3 & $3 / \mathrm{Cl}$ & 1 & & & & & 2 & & $3 / \mathrm{Cl}$ & 2 \\
\hline
\end{tabular}

Abb. 5: Zweite Repertorisation zu Simon [1].

\section{Repertoristion, Interpretation, Mittelgabe und Verlauf}

Zehn Arzneimittel decken alle Symptome ab, aber nur zwei davon haben keine Kontraindikationen. Erste Wahl ist Pulsatilla (PD 16), zweite Lycopodium (PD 14).

Mit Pulsatilla C 200 bessern sich Heuschnupfen und Asthma innerhalb von wenigen Tagen um $90 \%$. Weitere Dosen (M, XM, LM, CM) in monatlichen Abständen beseitigen die Atopie vollständig und bleibend. Beobachtungszeit: 5 Jahre.

\section{Fallbeispiel 2}

Die dreijährige Celine ist ein hypotones, hellhäutiges und schüchternes Kind, das seit der Säuglingszeit an einer generalisierten Neurodermitis leidet. Gelenkbeugen und Hautfalten sind besonders stark betroffen. Bisher wurde sie phasenweise mit Corticosteroiden behandelt, welche immer eine schnelle Besserung bewirkten. Der Ausschlag trat aber nach dem Absetzen immer wieder auf, weshalb die Eltern schließlich zu uns kommen. Sie schwitzt stark und kratzt sich oft blutig. Als „Nebenleiden“ hat sie mehrmals pro Jahr Atemwegsinfekte mit Fließschnupfen und trockenem Husten. Nachts knirscht sie mit den Zähnen. Vom Aussehen her denkt man bei diesem Kind an Calcium carbonicum. Dies darf aber in der Praxis nicht zu einer übereilten Verordnung ohne Beachtung evtl. weite- rer vorhandener wichtiger Symptome führen. Wir kommen nicht um eine richtige Fallaufnahme herum.

Mit den Fragebögen für Atemwegserkrankungen und Nebensymptome bereiten sich die Eltern auf die Fallaufnahme vor. Sie haben Folgendes beobachtet:

•• < beim Erwachen

$\bullet>$ Wärme-P

$\bullet<$ Einhüllen-P

$\bullet>$ im Freien-P

$\bullet>$ Ruhe-P

$\bullet>$ Liegen-P

-. Durst-P

-. Speichelvermehrung-P

- Verlangen freie Luft-P (bei Husten)

•. Verlangen nach Bewegung-P (bei Husten)

Viele Kinder haben ein Verlangen nach freier Luft und nach Bewegung. Diese besitzen nur einen Wert als Symptom, wenn sie bei Krankheit verstärkt sind. Man muss sich also unbedingt versichern, dass dem so ist. Die Mutter bestätigt dies. Besserung durch Ruhe ist das Gegenteil von Besserung durch Bewegung. Auch hier ist die Rückfrage wichtig, weil das Kind ja ein gesteigertes Verlangen nach Bewegung hat. Sie bestätigt auch das, so dass wir alles für die Repertorisation verwenden können. 
C. B.

Neurodermitis

\begin{tabular}{|c|c|c|c|c|c|c|c|c|c|c|c|c|c|c|}
\hline & Bonx. & Bry. & Acon. & calc. & Sulph. & Verat. & Staph. & Rhus. & Lyc. & Sep. & Mur-ac. & Arn. \\
\hline \multicolumn{3}{|l|}{ Hits } & 10 & 10 & 10 & 10 & 10 & 10 & 10 & 10 & 10 & 10 & 10 & 9 \\
\hline \multicolumn{3}{|c|}{ Sums } & 20 & 25 & 20 & 23 & 23 & 19 & 19 & 24 & 21 & 17 & 12 & 23 \\
\hline \multicolumn{3}{|c|}{ Polarity Difference } & 12 & 10 & 10 & 8 & 7 & 6 & 6 & 2 & 0 & -8 & -4 & 12 \\
\hline 111 & <Beim Erwachen & $P$ & 1 & 2 & 1 & 4 & 5 & 2 & 3 & 4 & 4 & 4 & 2 & 3 \\
\hline 90 & >Wärme allg. & $\mathbf{P}$ & 3 & 2 & 3 & 1 & 3 & 1 & 2 & 4 & 1 & 2 & 2 & 2 \\
\hline 37 & $<$ Warmeinhüllen & $\mathbf{P}$ & 3 & 1 & 3 & 3 & 2 & 3 & 2 & 1 & 4 & 1 & 1 & \\
\hline 93 & $>$ Im Freien & $P$ & 2 & 2 & 3 & 1 & 2 & 2 & 1 & 1 & 2 & 1 & 1 & 2 \\
\hline 76 & Verlangen nach freier Luft & $\mathrm{P}$ & 3 & 1 & 1 & 1 & 1 & 1 & 1 & 1 & 3 & 1 & 1 & 3 \\
\hline 58 & Verlangen nach Bewegung & $P$ & 1 & 2 & 2 & 1 & 1 & 2 & 1 & 4 & 1 & 1 & 1 & 3 \\
\hline 117 & > Ruhe (> nicht Bewegen) & $P$ & 2 & 4 & 1 & 2 & 1 & 1 & 3 & 1 & 1 & 1 & 1 & 3 \\
\hline 106 & $>$ Liegen & $\mathrm{P}$ & 1 & 4 & 1 & 3 & 1 & 1 & 2 & 1 & 1 & 1 & 1 & 3 \\
\hline 99 & Durst & $P$ & 2 & 4 & 4 & 4 & 4 & 3 & 1 & 3 & 1 & 2 & 1 & 3 \\
\hline 117 & Speichelvermehrung & $\mathbf{P}$ & 2 & 3 & 1 & 3 & 3 & 3 & 3 & 4 & 3 & 3 & 1 & 1 \\
\hline 28 & > Erwachen, beim & & & 1 & & 1 & & & & & & 4 & & \\
\hline 73 & <Wärme allg. & & 1 & 1 & 1 & 1 & 2 & 1 & 1 & 1 & 2 & 1 & 1 & 1 \\
\hline 56 & > Warmeinhüllen & & 1 & 1 & 1 & & & & 2 & $4 / C I$ & & 2 & 1 & 2 \\
\hline 110 & $<$ Im Freien & & 1 & 1 & & 2 & 1 & 1 & 2 & 2 & 1 & 1 & 2 & 1 \\
\hline 86 & Abneigung gegen freie Luft & & & $3 / \mathrm{Cl}$ & & $4 / C I$ & $3 / \mathrm{Cl}$ & 1 & 2 & $3 / \mathrm{Cl}$ & 3 & $3 / \mathrm{Cl}$ & 2 & 1 \\
\hline 68 & Abneigung gegen Bewegung & & 1 & 2 & $4 / \mathrm{Cl}$ & 1 & 1 & & & & $3 / \mathrm{Cl}$ & 2 & 1 & 1 \\
\hline 102 & $<$ Ruhe, in der & & 1 & 1 & 1 & 1 & 1 & 2 & 1 & $4 / \mathrm{Cl}$ & $4 / \mathrm{Cl}$ & $3 / \mathrm{Cl}$ & 2 & 1 \\
\hline 125 & $<$ Liegen & & 2 & 1 & 1 & 1 & 2 & 2 & 1 & $4 / \mathrm{Cl}$ & $4 / \mathrm{Cl}$ & $3 / \mathrm{Cl}$ & $3 / \mathrm{Cl}$ & 1 \\
\hline 86 & Durstlosigkeit & & & 1 & & 1 & 2 & 2 & $3 / \mathrm{Cl}$ & 2 & 1 & $3 / \mathrm{Cl}$ & 2 & 1 \\
\hline 111 & Speichelverminderung & & 1 & 3 & 2 & 3 & $4(C I)$ & $4(\mathrm{Cl})$ & 1 & 2 & 3 & 3 & 2 & 2 \\
\hline
\end{tabular}

Abb. 6: Repertorisation zu Celine [1].

\section{Repertorisation, Interpretation und Materia- medica-Abgleich}

Elf Arzneimittel decken alles ab - Borax hat als einziges keine Kontraindikationen. Bryonia und Aconitum kämen aufgrund der hohen Polaritätsdifferenz zusätzlich infrage, aber die Mutter bestätigt die Symptome „Verlangen nach freier Luft“ und „Verlangen nach Bewegung“, die die Kontraindikationen für diese Mittel begründen. Das Symptom „Zähneknirschen“ ist im Therapeutischen Taschenbuch nicht vorhanden. Wir suchen es deshalb in C.M. Bogers Boenninghausens Characteristics and Repertory: Teeth grinding: Borax, Aconitum (u.a.) [4: 422].

Borax [12]:

Starkes Jucken auf den Gelenken der Fingerrücken, daß er heftig kratzen muß. Rote Ausschlagblüten auf den Wangen und um das Kinn, [beim Säugling]. Flechtenausschlag auf dem Hinterbacken des Kindes.

\section{Mittelgabe und Verlauf}

Celine erhält eine Dosis Borax C 30.

Nach einer kurzen Verschlimmerung bessert sich die Haut während zwei Wochen um $80 \%$, danach wird der Ausschlag wieder stärker. Mit Borax C 200 steigt die Bes- serung auf $90 \%$, und Borax M bringt die Neurodermitis zum Verschwinden. Gleichzeitig hört auch das Zähneknirschen auf. - Vier Wochen später trinkt das Kind an einem heißen Sommertag Pfefferminzsirup, und der Hautausschlag flackert nochmals kurz auf. Borax XM löst das Problem definitiv und bringt auch die inneren Symptome völlig zum Verschwinden. Keine weiteren Mittelgaben. Beobachtungszeit: 5 Jahre.

\section{Diskussion}

Das erste Fallbeispiel demonstriert die Schwierigkeiten der Neurodermitis-Behandlung bei Säuglingen anschaulich. Zunächst scheitert der Versuch mit den bewährten Indikationen Belladonna und Sulfur, und Impfnosoden kommen nicht infrage, weil das Kind noch nie geimpft wurde. Da innere Symptome fehlen, machen wir die Fallaufnahme nur mit Hautsymptomen. Graphites bessert die Erkrankung, es fällt aber auf, dass Simons Haut immer noch irritabel und damit nicht völlig unter Kontrolle ist. Nach der Scharlacherkrankung und Aconitum verlagert sich die Symptomatik in die Tiefe. Der Verlauf ähnelt einer Unterdrückung von Hautsymptomen mit Cortison. Jetzt haben wir aber innere Symptome zur Verfügung und finden damit das Arzneimittel, das alles heilt. 
Was hätte es gebraucht, um direkt auf Pulsatilla zu stoßen? Pulsatilla stand in der ersten Repertorisation an 9. Stelle, hatte aber zwei Kontraindikationen (< Kälte, $<$ Bewegung). Kleine Säuglinge sind an sich kälteempfindlich, so verstand die Mutter die Frage; aber das ist normal, also kein Symptom. Wir hätten es weglassen können. Unter „Bewegung bessert“ kann in diesem Fall nur passive Bewegung, also „Herumtragen bessert“ gemeint sein. Auch das ist kein Symptom, weil es dabei eher um Zuwendung und Trost geht. „Sonne verschlimmert“ könnte auch bedeuten, dass „Wärme verschlimmert“. Ändern wir die Symptomatik in dieser Weise, so ist Pulsatilla unter den 4 besten Mitteln.

Das sind Überlegungen, die wir nachträglich machen können, weil wir mehr wissen. Im akuten Fall kann es bei Säuglingen sehr schwierig sein, Symptome von Eigenheiten zu unterscheiden. Viel einfacher war es bei der zweiten Patientin, die sich bereits mit inneren Symptomen präsentierte.

Bestimmen wir bei Neurodermitis-Patienten das homöopathische Mittel nur mit Hautsymptomen, so beträgt die Erfolgsrate ungefähr $30 \%$. Können innere Symptome zur Mittelbestimmung herangezogen werden, so steigt sie auf über $60 \%$ (unveröffentlichte Resultate). Nur können wir bei Säuglingen nicht einfach warten, bis sie innere Symptome entwickeln...

Die Polaritätsanalyse leistet einen wertvollen Beitrag zur Heilung, weil sie die Mittelfindung bei oligosymptomatischen Fällen oft sehr erleichtert, manchmal überhaupt erst ermöglicht.

\section{Online zu finden unter:}

http://dx.doi.org//10.1055/s-0042-112508

\section{-. Literatur}

[1] http://polarity-analysis.com

[2] Boenninghausen Cv. Aphorismen des Hippokrates. Göttingen: Burgdorf; 1979. S. 407.

[3] Boenninghausen Cv. Boenninghausens Therapeutisches Taschenbuch. Revidierte Ausgabe. Hrsg. Gypser KH: Stuttgart: Sonntag; 2000.

[4] Boger CM. Boenninghausens Characteristics and Repertory. Reprint; New Delhi: Jain Publishers; 1984.

[5] Frei H. Treatment of Hyperactive Children: Increased Efficiency through Modifications of Homeopathic Diagnostic Procedure. Homeopathy 2006; 95: 163-170.

[6] Frei H. Polarity Analysis, a New Approach to Increase the Precision of Homeopathic Prescriptions“. Homeopathy 2009; 98: 4955.

[7] Frei H. Die Polaritätsanalyse in der Homöopathie - Ein präziser Weg zum homöopathischen Arzneimittel. Kandern: Narayana; 2014.

\section{Dr. med. Heiner Frei \\ Spezialarzt FMH für Kinder und Jugendliche \\ Kreuzplatz 6 \\ CH 3177 Laupen}

Heiner Frei ist Facharzt für Kinder und Jugendliche, speziell pädiatrische Homöopathie in Laupen bei Bern. Lehrtätigkeit in der Schweiz und international seit 1994. Wissenschaftliche Publikationen u.a. über akute Tonsillitis, Otitis media, ADS/ADHS, H1N1-

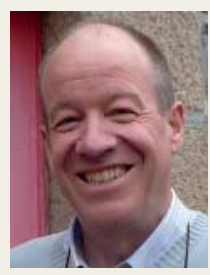

Grippe, multimorbide Patienten und homöopathische Methodik. 2001-2005 Studienleiter der Berner ADHS-Doppelblindstudie, die einen rigorosen Nachweis der Wirkung homöopathischer Arzneimittel erbrachte. Seit 2001 Entwicklung der Polaritätsanalyse und Veröffentlichung mehrerer Bücher zu diesem Thema (ADHS, akute und chronische Erkrankungen, multimorbide Patienten). Preisträger der Max Tiedemann-Stiftung 2009.

[8] Frei H. Homeopathic Treatment of Multimorbid Patients: A Prospective Outcome Study with Polarity Analysis. Homeopathy 2015; 104: 57-65.

[9] Frei H, Everts R, Ammon Kv et.al.. Homeopathic Treatment in Children with Attention Deficit Hyperactivity Disorder - A Randomized, Double-Blind, Placebo-Controlled Crossover Trial. European Journal of Pediatrics 2005; 164: 758-767.
[10] Hahnemann S. Organon der Heilkunst. 6. Auflage. Hrsg. Schmidt JM, Stuttgart: Haug; 2002.

[11] Hering C. Hahnemanns Three Rules Concerning the Rank of Symptoms. Hahnemannian Monthly, August 1865; 5-12.

[12] Hering C. The Guiding Symptoms of our Materia Medica. Reprint; New Delhi: B. Jain Publishers; 1991 [1879]. 